\title{
Turbulent transport of energetic ions
}

\author{
T. Dannert $^{1}$, S. Günter ${ }^{2}$, T. Hauff ${ }^{2}$, F. Jenko ${ }^{2}$ \\ ${ }^{1}$ Ecole Polytechnique Fédérale de Lausanne (EPFL), Centre de Recherches en Physique \\ des Plasmas, Association Euratom-Confédération Suisse, CH-1015 Lausanne, \\ Switzerland \\ ${ }^{2}$ Institut für Plasmaphysik, EURATOM-Assoziation, Boltzmannstr. 2, D-85748 \\ Garching, Germany
}

Anomalous transport of heat and particles is known to be a key issue for fusion devices. In the present paper the anomalous transport of fast ions is investigated. High transit frequencies of the fast ions lead to the assumption that there is only very weak interaction with the low-frequency turbulence. However, our results indicate a significant fast ion particle transport due to the turbulence. To investigate this interaction, a second ion species is introduced into a saturated gyrokinetic turbulence simulation and the transport effects of the turbulence on the fast ions is analyzed. The fast ion species can serve as a model for ions which are introduced by neutral beam injection (NBI).

The simulation of the turbulence and the fast ions is done with the GENE $[1,2]$ code. The beam ions are treated as passive tracers but obey the fully gyrokinetic dynamics. As model for the equilibrium beam ion distribution an unsymmetric Maxwellian with two different temperatures in beam direction and in counter-beam direction is used. This model and the resulting modifications to the gyrokinetic equations are discussed. The influence of the turbulence on the particle distribution is presented, together with the dependencies on beam parameters. A link to the twodimensional studies of Hauff and Jenko [3] is established and a physical picture of the processes involved will be shown.

The presented results have an impact on the NBI current drive and are a possible explanation for results reported from Asdex Upgrade [4], where the decrease of the current drive efficiency with higher input power was explained by a turbulent redistribution of fast ions. Future experiments, which make extensive use of NBI current drive, may also be affected by this turbulent particle transport of energetic ions.

\section{References}

[1] F. Jenko et al., Phys. Plasmas 7 (2000), 1904

[2] T. Dannert and F. Jenko, Phys. Plasmas 12 (2005), 072309

[3] T. Hauff and F. Jenko, Phys. Plasmas 13 (2006), 102309

[4] S. Günter et al., 31st EPS Conference on Plasma Phys. London (2004) 